\title{
Epicardial Termination of Left Atrial Appendage Atrial Tachycardia
}

\author{
MARCO POLSELLI, MD, ${ }^{1}$ FILIPPO M. CAUTI, MD, ${ }^{1}$ PIETRO ROSSI, MD, PhD, ${ }^{1}$ RICCARDO MADDALENA, MD, ${ }^{1}$ \\ and STEFANO BIANCHI, MD ${ }^{1}$
}

${ }^{1}$ Arrhythmology and Electrophysiology Unit, Ospedale San Giovanni Calibita, Fatebenefratelli Hospital, Isola Tiberina, Rome, Italy

\begin{abstract}
This case report describes a third successful attempt to ablate a focal atrial tachycardia originating from the left atrial appendage in a highly symptomatic 49-year-old woman using a combined endocardial-epicardial approach, which could be taken into consideration as a safe and effective alternative method for treating similar arrhythmias originating from complex sites.
\end{abstract}

KEYWORDS. Case report, epicardial ablation, left atrial appendage tachycardia.
ISSN 2156-3977 (print) ISSN 2156-3993 (online) CC BY 4.0 license

(C) 2021 Innovations in Cardiac Rhythm Management

\section{Case presentation}

A 49-year-old woman with a history of incessant atrial tachycardia (AT) was referred to our arrhythmia unit. She was diagnosed with focal AT at the age of 17 years and treated with different classes of antiarrhythmic drugs, without a significant burden reduction. She was found to be intolerant to flecainide because of vision disturbances. Therefore, $160 \mathrm{mg}$ /day of sotalol was prescribed, which she continued to take despite experiencing hypotension and asthenia as side effects.

After multiple electrical cardioversions, she was referred for a first ablation attempt at another center. The activation map revealed a left atrial appendage (LAA) focal AT, with a cycle length (CL) of $485 \mathrm{~ms}$ (124 bpm). Radiofrequency (RF) ablation was performed from the endocardium, near the entrance of the LAA, without leading to AT termination. She was thus rescheduled for cryoablation in another center, in order to avoid LAA perforation. A second attempt was made with the use of a cardiac cryoablation catheter (Freezor; Medtronic, Minneapolis, MN, USA), which led only to a slowing of the arrhythmia,

The authors report no conflicts of interest for the published content. Manuscript received January 9, 2021. Final version accepted April 28, 2021.

Address correspondence to: Marco Polselli, MD, Arrhythmology Unit, Ospedale San Giovanni Calibita, Fatebenefratelli, Via Ponte Quattro Capi 39, 00186 Rome, Italy. Email: marco.polselli@live.it. without termination. The patient was then referred to our department for re-evaluation.

The admission electrocardiogram (ECG) showed an incessant AT with a CL of $548 \mathrm{~ms}$ (109 bpm), with negative P-waves in D1 and positive P-waves in DII, DIII, and precordial leads (Figure 1). Cardiac magnetic resonance imaging was performed to study the shape of the LAA and its anatomical details, which demonstrated a "chicken wing" morphology. A new ablation procedure was scheduled with a combined endocardial-epicardial approach.

Under general anesthesia, a transesophageal echocardiography probe was positioned to rule out LAA thrombi, monitor catheter positions, and control RF delivery. Before systemic anticoagulation, inferior epicardial access was obtained to target epicardially the region of interest via a steerable sheet (Agilis EPI; Abbott) (Figure 2A). The activated clotting time was maintained between 300 and 350 seconds. A high-density electroanatomic map (EAM) of the epicardial layer above the LAA was obtained via a multipolar high-density catheter (HD Grid; Abbott, Chicago, IL, USA) (Figures 2B and 2C). High output pacing and tag positioning on the EAM outlined the phrenic nerve course.

A left atrial endocardial EAM was obtained using the HD Grid, via a patent foramen ovale, with the catheter's soft, closed shape permitting gentle reconstruction of the LAA endocardial anatomy. The LAA EAM was then refined via 


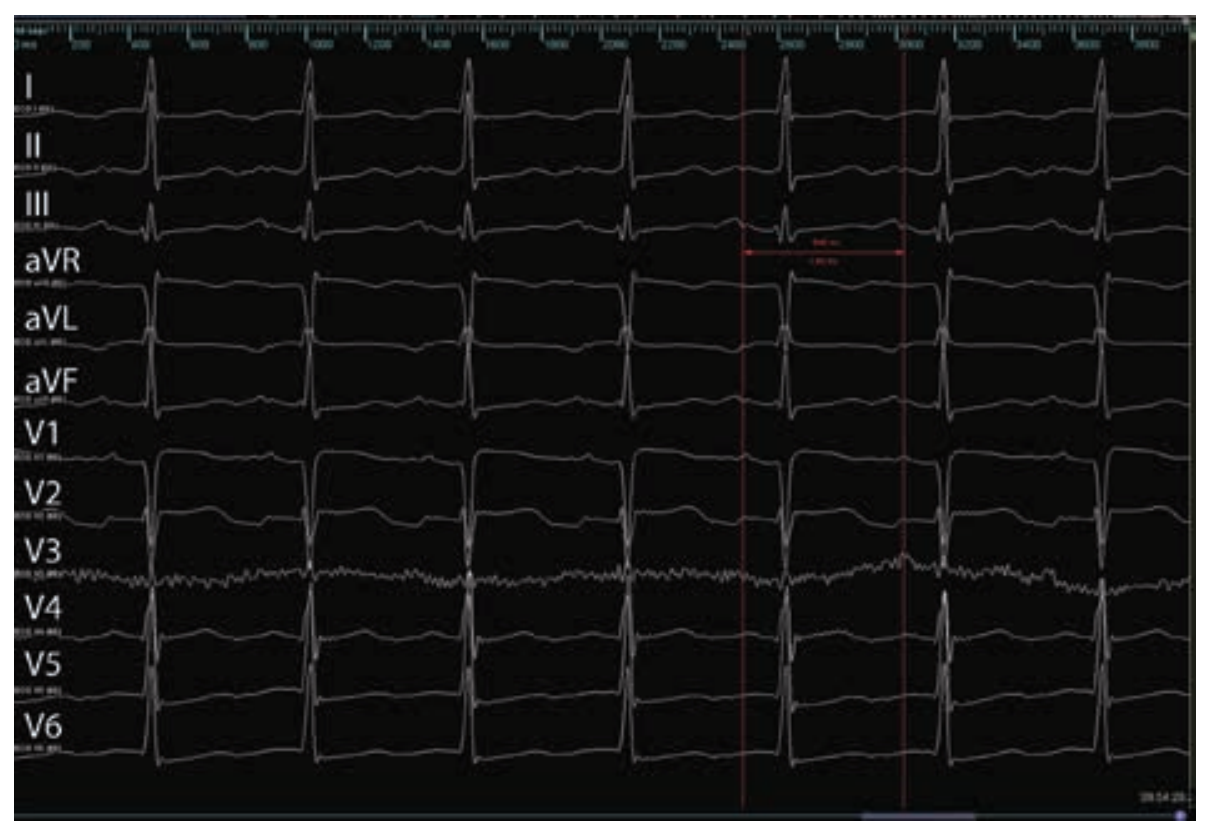

Figure 1: Baseline ECG showing the clinical atrial tachycardia, with a CL of $548 \mathrm{~ms}$ (109 bpm). Negative P-waves in D1 and positive P-waves in DII, DIII, and precordial leads.

A

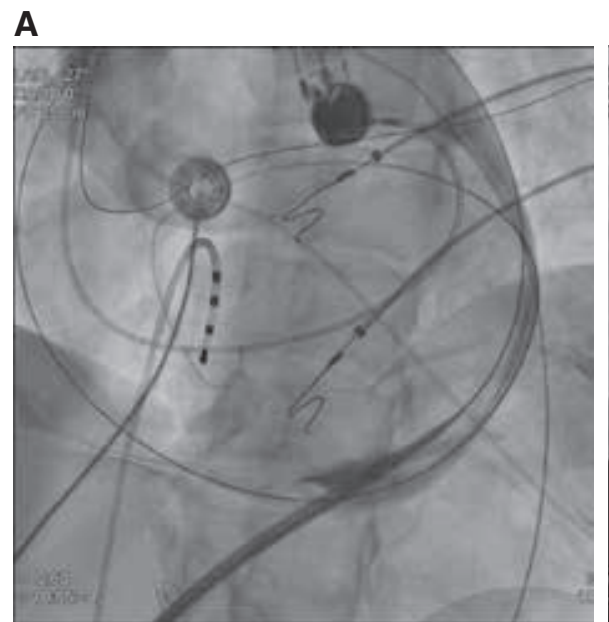

C

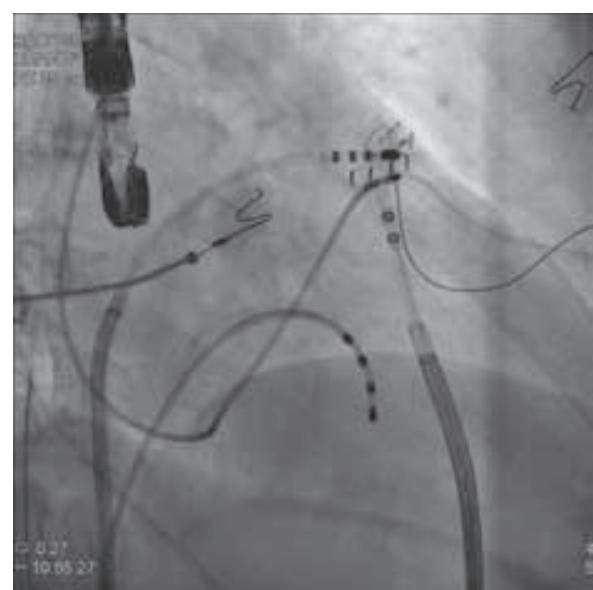

B

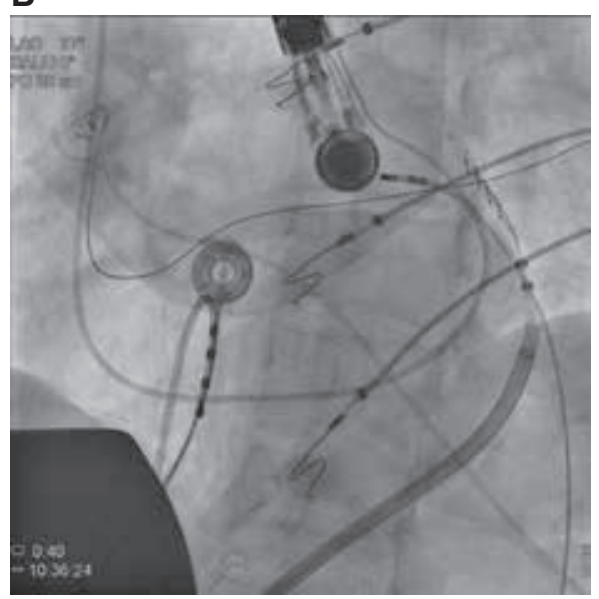

D

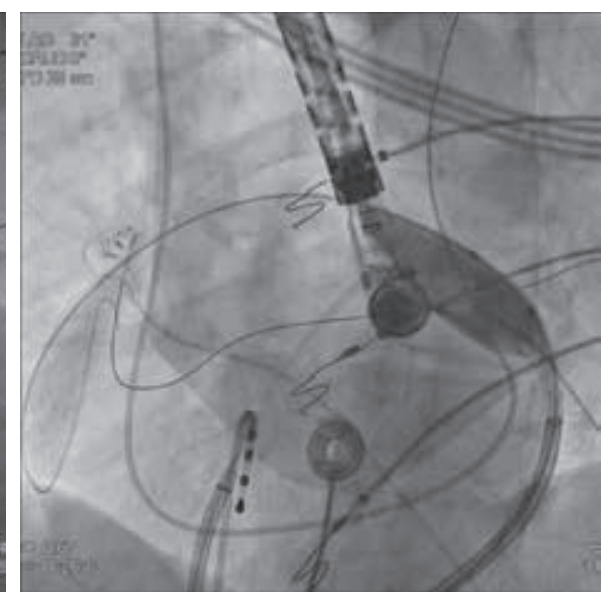

Figure 2: A: Epicardial access confirmed via dye injection. B and C: Left and right anterior oblique fluoroscopic views of the LAA and catheter positioning. D: Epicardial balloon inflated to displace the phrenic nerve during ablation. 


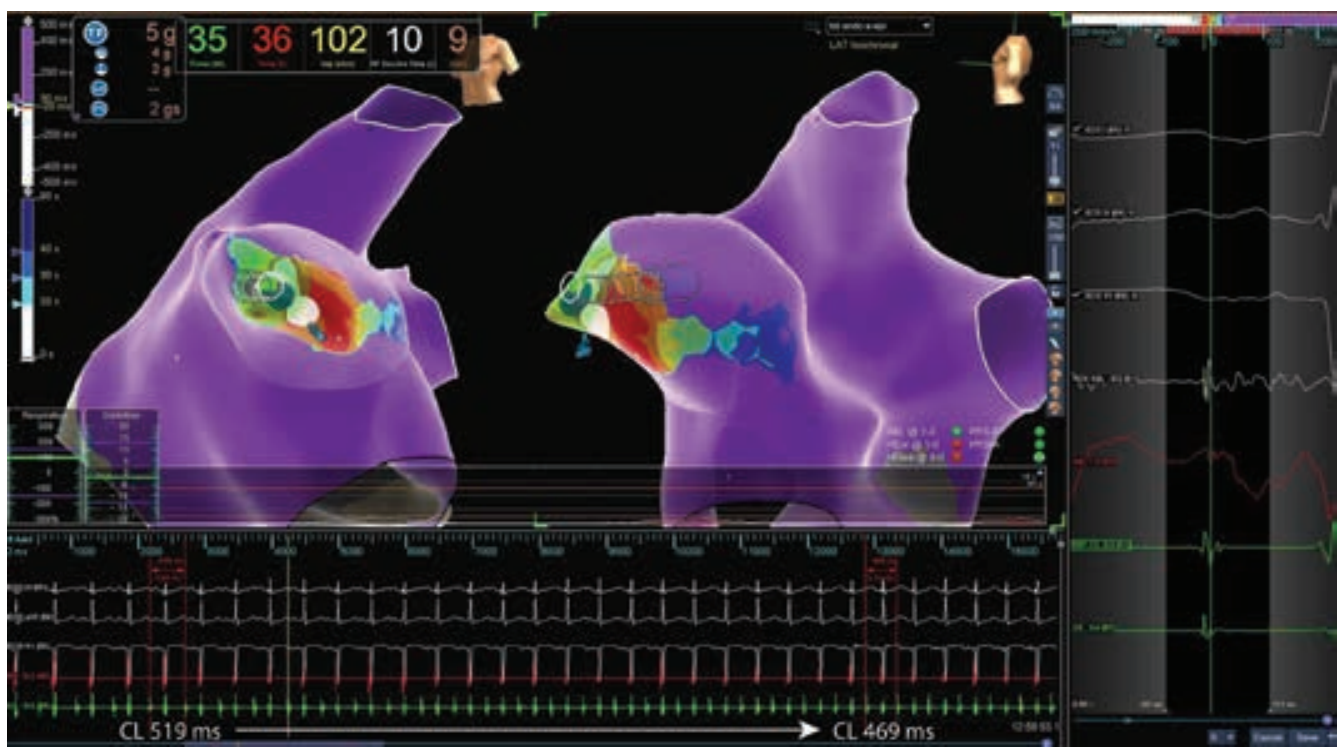

Figure 3: An endocardial attempt with arrhythmia warm-up.

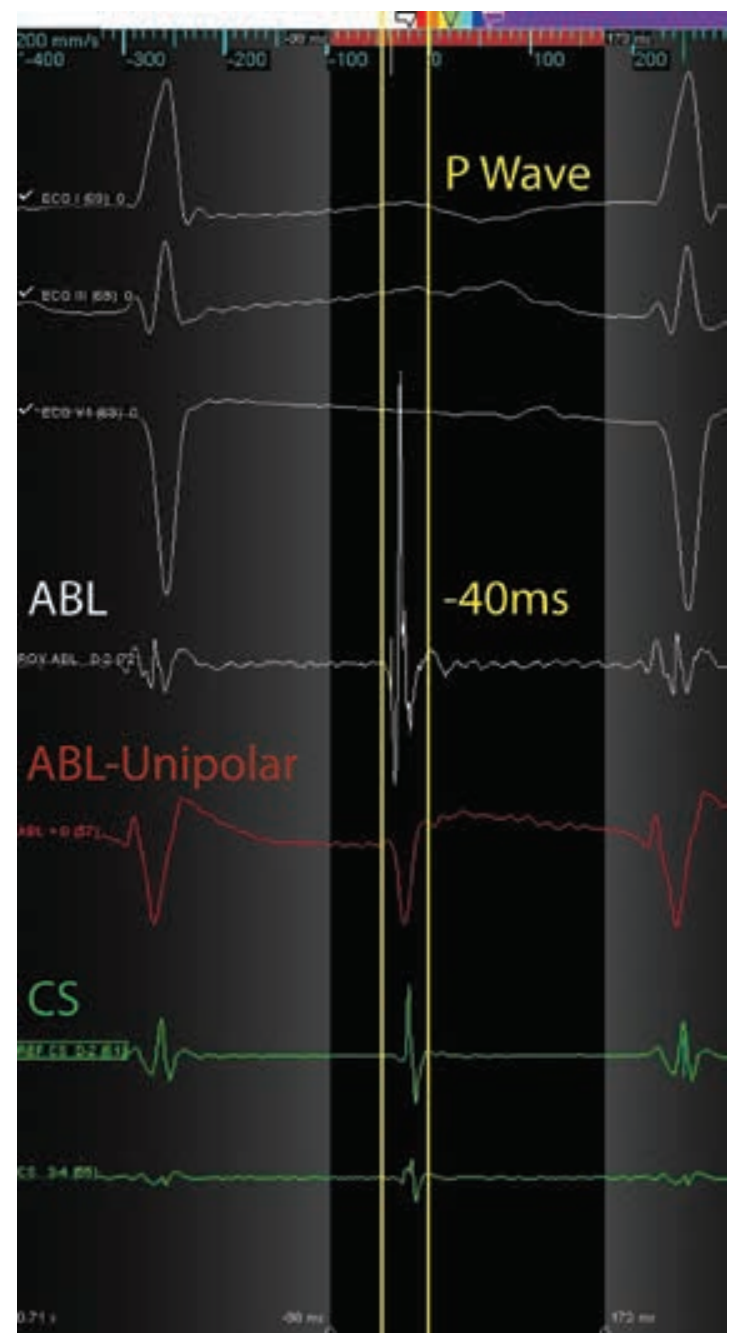

Figure 4: Epicardial EGM recorded by the ablation catheter. Unipolar EGM with QS activation. ABL: ablation; CS: coronary sinus; ECG: electrocardiogram. an optical fiber, contact-sensing, open-irrigated ablation catheter (TactiCath SE, Sensor Enabled; Abbott), which was constantly monitored via transesophageal echocardiography visualization.

Two endocardial RF applications were performed with $35 \mathrm{~W}$ at $43^{\circ} \mathrm{C}$ at the earliest electrogram (EGM) after phrenic nerve displacement via an epicardial balloon (Figure 2D). The RF application influenced the arrhythmia cycle by warming it up (CL shortened), without interrupting it (Figure 3). A second attempt was then made epicardially, during phrenic nerve displacement via a second epicardial access, at the earliest EGM ( $-40 \mathrm{~ms})$, slightly lower and more anterior in respect to the endocardial ablation site (Figure 4). A single RF pulse at $40 \mathrm{~W}$ and $43^{\circ} \mathrm{C}$ (15 g of tangential force) terminated the arrhythmia after seven seconds (Figure 5).

An intrapericardial corticosteroid was injected as per the center's protocol at the end of the procedure and a pigtail catheter was left in the left lateral epicardial wall. No complication occurred. Epicardial drainage was removed three hours after the procedure. No more arrhythmias occurred during the hospital stay. The patient was discharged the day after the procedure without antiarrhythmic drugs. Considering the pinpoint endocardial application and the LAA filling/voiding speeds, anticoagulation therapy was maintained for one month after the ablation procedure.

At three months of follow-up, a 24-hour Holter ECG was performed, which showed the absence of arrhythmias, excluding a small number of isolated premature atrial complexes. The patient was completely asymptomatic and reported a drastic improvement in her quality of life.

\section{Discussion}

The LAA is among the specific anatomic structures that can be responsible for AT originating from the left atrium. 


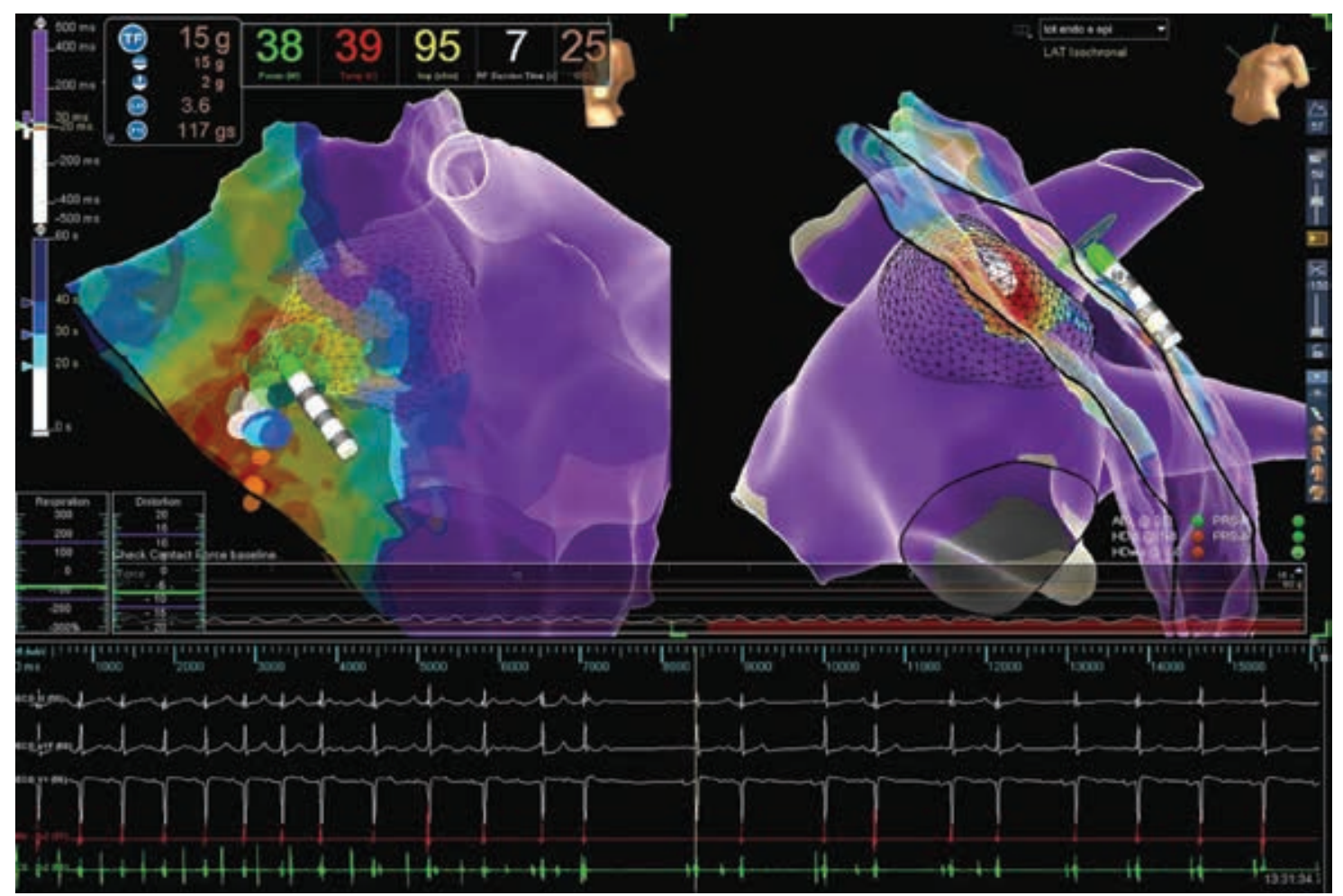

Figure 5: Epicardial interruption during RF of the clinical tachycardia. Ablation parameters: power of $38 \mathrm{~W}$, temperature of $39^{\circ} \mathrm{C}$.

This structure originates from the embryonic left atrium ${ }^{1}$ and usually has an elongated, finger-like structure with different shapes forming its body ("chicken wing," "cactus-like," "cauliflower," and "windsock") ${ }^{2}$ differently from the right atrial appendage, which has a more constant, triangular shape. The LAA is positioned between the left superior pulmonary vein, the left circumflex artery, and the great cardiac vein. The LAA anatomy, with different shapes; pectinate muscles; and the fragile, thin myocardium being among its characteristics, poses some serious challenges to the electrophysiologists approaching this structure, making it difficult to easily reach the distal part of the appendage with catheters or making it risky to ablate with sufficient energy inside this structure.

ECG patterns have been developed ${ }^{3-6}$ to identify the specific site of origin of a focal tachycardia, but the targeting capability of a modern high-definition mapping system overcomes most of the uncertainties about the exact electroanatomic source of the tachycardia, guiding the operator to the precise site of origin to target for ablation.

Our case report underlines the relevance of these little "gaps" in the precise localization of the ectopy inside particular and anatomically complex structures, where ablation could prove to be too dangerous and, in some cases, harmful due to the low thickness of the myocardium. In such cases, where ectopy arises from the LAA, an epicardial approach could be the only way to reach and effectively ablate the source of the arrhythmia, when different ablation strategies fail (endocardial RF and endocardial cryo in our case), as reported in a few previous experiences. ${ }^{78}$ Moreover, for this same reason, an epicardial approach could prove counterintuitively safer than an endocardial approach, limiting the power and application time to those strictly necessary to ablate the ectopic focus, thus preserving the integrity of the surrounding myocardium and structures.

In our case, we were able to map and then displace the phrenic nerve with a balloon inflated in the pericardium, avoiding contact between the ablating catheter and the nerve, thereby eliminating the chance of palsy.

Different approaches for LAA tachycardia ablation have been proposed, such as cryoballoon LAA isolation, ${ }^{9}$ LAA resection with thoracoscopic or video-assisted thoracoscopic surgery, ${ }^{10,11}$ or device-based approaches (eg, Atriclip; Atricure, Mason, $\mathrm{OH}$, USA ${ }^{12}$ or Lariat; SentreHEART, Redwood City, CA, USA ${ }^{13}$ ), all with relevant potential risks (eg, staple line failure).

An endocardial-epicardial approach could also help in reducing the hospital stay and the associated costs.

In terms of safety, a confined, short RF application should not reduce LAA systolic function or total blood flow, as previously demonstrated. . $^{514}$

\section{Conclusions}

Epicardial ablation is an effective and safe approach to ablate focal tachycardias originating from the LAA, particularly from the distal part of it, which can be difficult 
to reach with an endocardial catheter, given the complex anatomy ${ }^{1}$ of the structure and the frailty of the myocardium at this level. This minimally invasive approach is probably safer than others, like appendage resection (risk of suture failing; furthermore, the LAA is not a useless structure), whole isolation of LAA with devices (presence of a foreign body), or cryoablation ${ }^{15}$ (possibility of clot formation and LAA contractility reduction), and could help to avoid ineffective and eventually harmful multiple endocardial attempts. ${ }^{16,17}$

\section{References}

1. Ho SY, Cabrera JA, Sanchez-Quintana D. Left atrial anatomy revisited. Circ Arrhythm Electrophysiol. 2012;5(1):220-228.

2. Al-Saady NM, Obel O, Camm AJ. Left atrial appendage: structure, function and role in thromboembolism. Heart. 1999;82(5):547-554.

3. Kistler PM, Roberts-Thomson KC, Haqqani HM, et al. P-wave morphology in focal atrial tachycardia development of an algorithm to predict the anatomic site of origin. J Am Coll Cardiol. 2006;48(5):1010-1017.

4. Kistler PM, Sanders P, Hussin A, et al. Focal atrial tachycardia arising from the mitral annulus: electrocardiographic and electrophysiologic characterization. J Am Coll Cardiol. 2003;41(12):2212-2219.

5. Yamada T, Murakami Y, Yoshida Y, et al. Electrophysiologic and electrocardiographic characteristics and radiofrequency catheter ablation of focal atrial tachycardia originating from the left atrial appendage. Heart Rhythm. 2007;4(10):1284-1291.

6. Wang YL, Li XB, Quan X, et al. Focal atrial tachycardia originating from the left atrial appendage: electrocardiographic and electrophysiologic characterization and long-term outcomes of radiofrequency ablation. J Cardiovasc Electrophysiol. 2007;18(5):459-464.

7. Phillips KP, Natale A, Sterba R, et al. Percutaneous pericardial instrumentation for catheter ablation of focal atrial tachycardias arising from the left atrial appendage. J Cardiovasc Electrophysiol. 2008;19(4):430-433.
8. Yamada T, McElderry HM, Allison JS, Kay GN. Focal atrial tachycardia originating from the epicardial left atrial appendage. Heart Rhythm. 2008;5(5):766-767.

9. Yorgun H, Canpolat U, Evranos B, Aytemir K. Entrapment of focal atrial tachycardia using cryoballoon ablation; sinus rhythm in the left atrium and ongoing atrial tachycardia in the left atrial appendage. Indian Pacing Electrophysiol J. 2017;17(6):189-191.

10. Torok RD, Wei B, Kanter RJ, Jaquiss RDB, Lodge AJ. Thoracoscopic resection of the left atrial appendage after failed focal atrial tachycardia ablation. Ann Thorac Surg. 2014;97(4):1322-1327.

11. Guo X-G, Zhang J-L, Ma J, et al. Management of focal atrial tachycardias originating from the atrial appendage with the combination of radiofrequency catheter ablation and minimally invasive atrial appendectomy. Heart Rhythm. 2014;11(1):17-25.

12. Benussi S, Mazzone P, Maccabelli G, et al. Thoracoscopic appendage exclusion with an atriclip device as a solo treatment for focal atrial tachycardia. Circulation. 2011;123(14):1575-1578.

13. Atoui M, Pillarisetti J, Iskandar S, Earnest M, Lakkireddy D. Left atrial appendage tachycardia termination with a LARIAT suture ligation. J Atr Fibrillation. 2015;8(4):1380.

14. Yang Q, Ma J, Zhang S, Hu J-Q, Liao Z-L. Focal atrial tachycardia originating from the distal portion of the left atrial appendage: characteristics and long-term outcomes of radiofrequency ablation. Europace. 2012;14(2):254-260.

15. Wei H-Q, Sun Q, Guo X-G, Yang J-D, Ma D. Successful ablation of focal atrial tachycardia originating from the left atrial appendage using 23-mm second-generation cryoballoon. Heart Rhythm Case Rep. 2019;5(6):325-328.

16. Hasegawa K, Miyazaki S, Tada H. Sequential unipolar endocardial and epicardial ablation for focal atrial tachycardia originating from the deep left atrial appendage. Europace. 2019;21(1):53.

17. Biase LD, Schweikert RA, Saliba WI, et al. Left atrial appendage tip: an unusual site of successful ablation after failed endocardial and epicardial mapping and ablation. J Cardiovasc Electrophysiol. 2010;21(2):203-206. 